\title{
Transanal Evisceration Caused by Rectal Laceration
}

\author{
Martínez Perez A, Torres Sánchez T, Richart Aznar JM, Martí Martínez E, Martínez Abad M \\ Servicio de Cirugía General y del Aparato Digestivo, Hospital Universitario Doctor Peset, Valencia, Spain
}

Transrectal evisceration caused by colorectal injury is an unusual entity. This pathology is more frequent in elderly patients and it is usually produced spontaneously. Rectal prolapse is the principal predisposing factor. An 81-year-old woman was taken to the hospital presenting exit of intestinal loops through the anus. After first reanimation measures, an urgent surgery was indicated. We observed the absence of almost every small intestine loop in the abdominal cavity; these had been moved to the pelvis. After doing the reduction, a 3 to $4 \mathrm{~cm}$ linear craniocaudal perforation in upper rectum was objectified, and Hartmann's procedure was performed. We investigated and knew that she frequently manipulate herself to extract her faeces. The fast preoperative management avoided a fatal conclusion or an extensive intestinal resection. Reasons that make us consider rectal self-injury as the etiologic factor are explained.

Keywords: Rectum, Abdominal injuries, Intestinal perforation

\section{INTRODUCTION}

Transrectal evisceration caused by colorectal injury is an unusual entity. Since its first description by Brodie in 1827, not more than 60 adult cases have been described [1]. This pathology is more frequent in elderly patients, and it usually occurs spontaneously. Among the predisposing factors, rectal prolapse (60\%-70\%) should be highlighted [2]; it is also related to the existence of abnormally deep rectovaginal and rectovesical pouches. In children, cases caused by abdominal traumatism, suction by depuration systems in swimming pools and impaling have been described $[3,4]$.

We have found no previous reports in the literature of a rectal self-inducted perforation caused by digitation.

\section{CASE REPORT}

We present the case of an 81-year-old woman without interesting

Received: January 5, 2013 • Accepted: September 13, 2013

Correspondence to: Martinez Perez A, M.D.

Servicio de Cirugía General y del Aparato Digestivo, Hospital Universitario

Doctor Peset, Avenida Gaspar Aguilar, 90 Valencia 46017, Spain

Tel: +34-961622505, Fax: +34-961622501

E-mail: aleix.martinez.perez@gmail.com

(C) 2014 The Korean Society of Coloproctology

This is an open-access article distributed under the terms of the Creative Commons Attribution NonCommercial License (http://creativecommons.org/licenses/by-nc/3.0) which permits unrestricted non-

commercial use, distribution, and reproduction in any medium, provided the original work is properly cited. medical precedents excepting a moderated cognitive deterioration (with partial dependence for activities of daily living). She was taken to the hospital by her relatives after they found her in the bathroom presenting with intestinal loops exiting through her anus. The presence of a rectal prolapse was not among her precedents (Fig. 1).

On the first exploration, we recognized an abdominal excavation, which was painless on palpation, and we observed many small intestine loops with a congestive aspect exiting through the anus. When the patient arrived, she was hypotensive and tachycardic. After the first resuscitation attempt, an urgent blood test was requested, and the results showed albumin, 3; C-reactive protein, $47.3 \mathrm{mg} / \mathrm{dL}$; and 19,700 leukocytes with neutrophilia, indicating an urgent need for surgery. The patient was taken to the operating room less than two hours after the beginning of the symptoms, and during the surgery, her hemodynamic condition remained the same.

For the surgery, the patient was placed in a modified lithotomy position, and two areas (abdominal and perineal) were prepared. After checking the difficulty of reducing the intestine exclusively through the anus, we performed an exploratory laparotomy, which revealed the absence of small intestine loops in the cavity; these had been moved to the pelvis and this was causing an important traction of the mesentery root. As a result, a manual reduction of the loops to the cavity through intraperitoneal traction was done; this was controlled from the perineal field. After the reduction had been completed, a 3 to $4 \mathrm{~cm}$ lineal craniocaudal perforation 


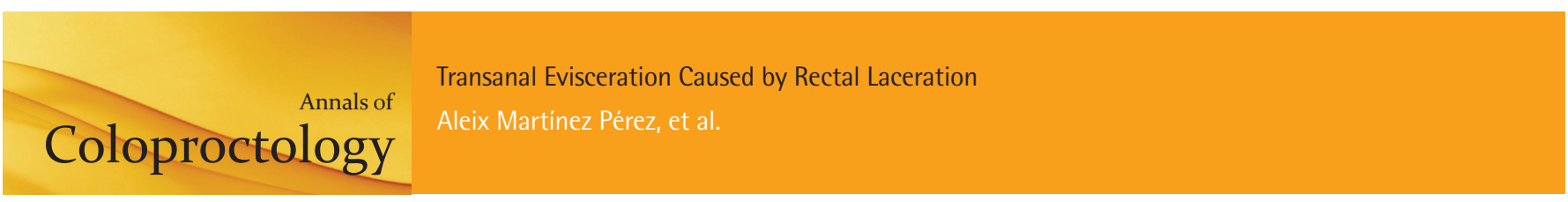

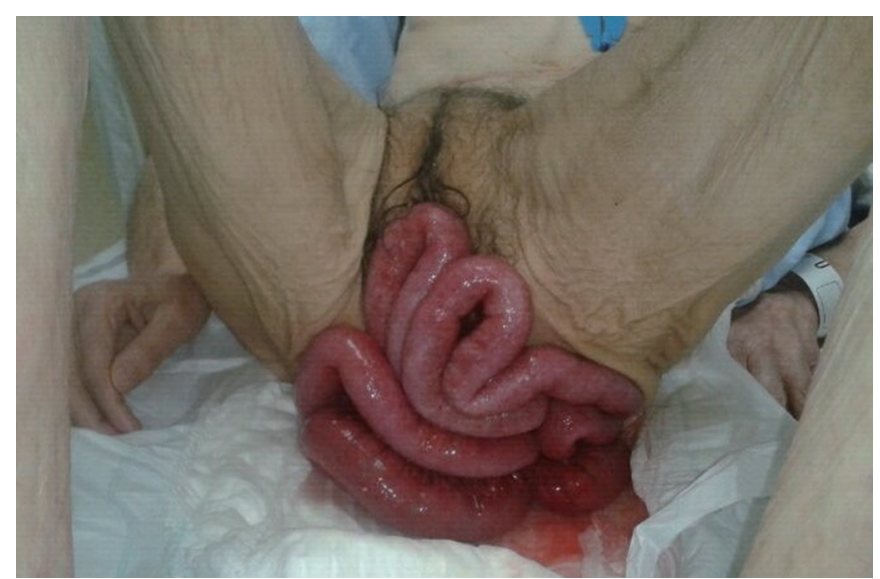

Fig. 1. Protrusion of several loops of the small bowel through the anus.

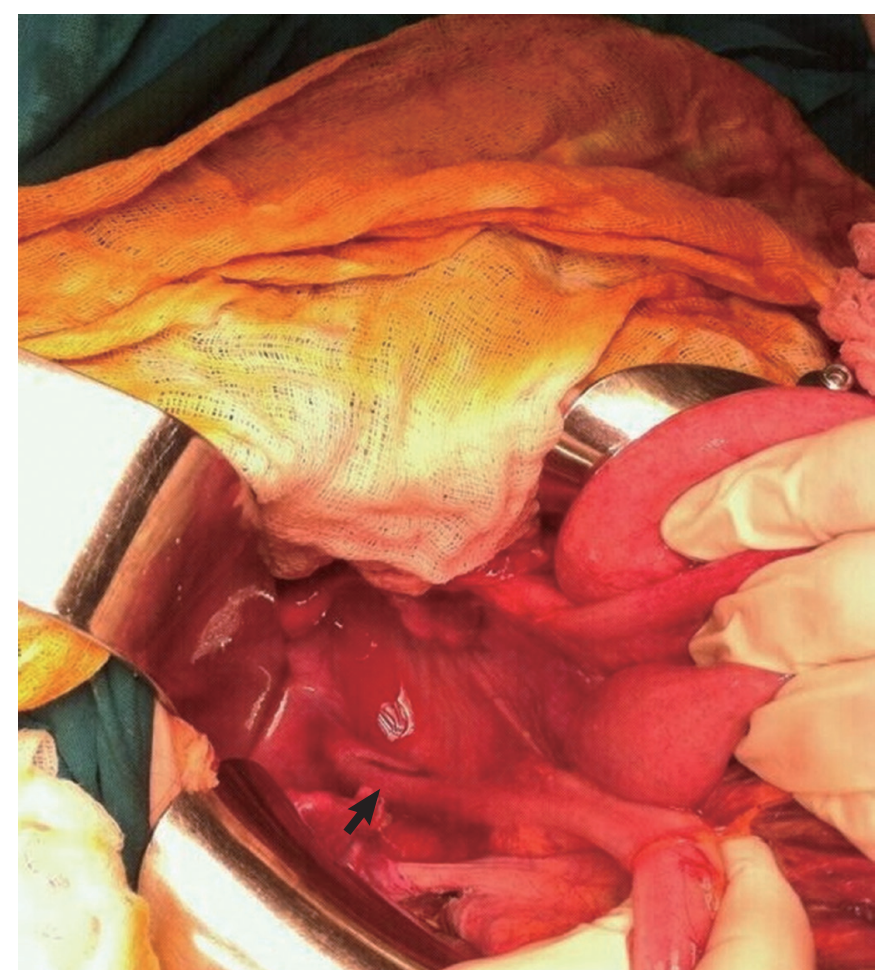

Fig. 2. Cranial view showing a longitudinal intraperitoneal rupture of the rectum and a deep rectovaginal pouch.

in the antimesenteric portion of the upper rectum was objectified (RIS II/AAC III). An important flaccidity of the entire pelvic musculature which contained a large rectovaginal pouch was observed, which resulted in both the superior rectum and the peritoneal reflection leaning on the pelvic floor (Fig. 2). The peritoneal cavity was abundantly washed with hot saline, and after some minutes, the loops initiated a visible peristalsis and took on a normal coloration. Later, the superior rectum and the sigmoid colon were dissected until the promontory, and an anterior resection of

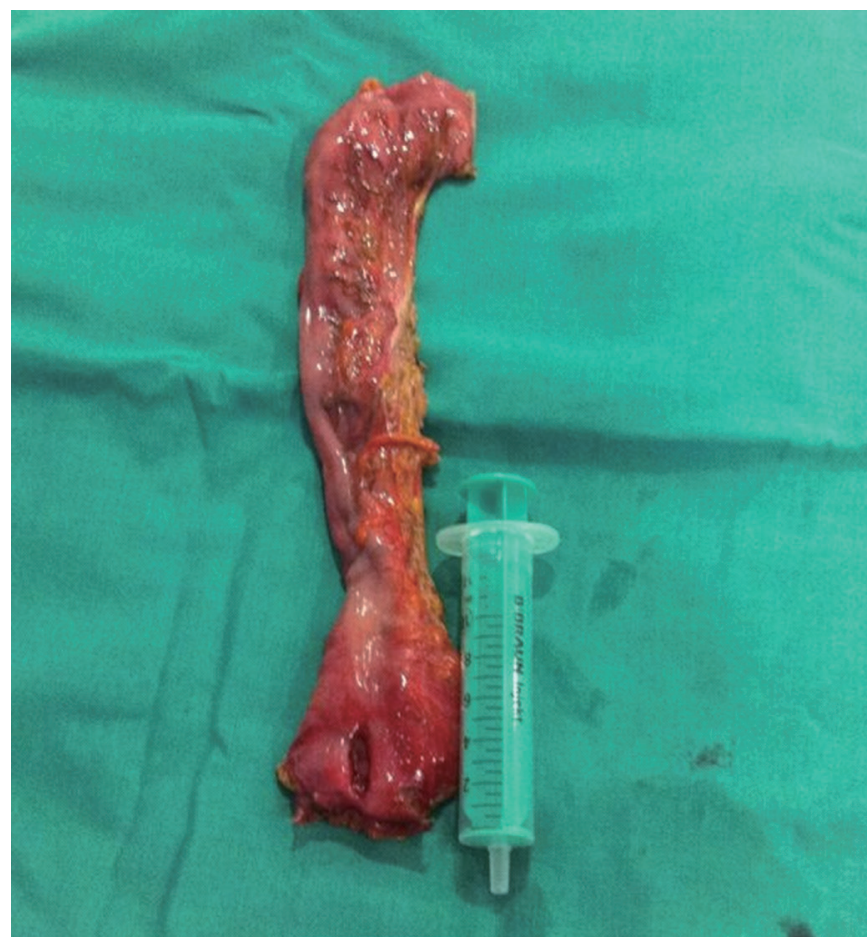

Fig. 3. Perforation in the antimesenteric portion of the upper rectum.

the rectum and a terminal colostomy in the left iliac region (Hartmann's procedure) were performed (Fig. 3).

Later, the patient's relatives told us that she had difficulties defecating and had frequently manipulate herself to extract her feces. The patient was admitted to the intensive care unit for 3 days where she remained hemodynamically stable and her ventilatory support was ceased without incidence. She had a slow recovery, and on the 6th day tolerated oral intake well. She was discharged after 13 days, and two months later remained asymptomatic.

\section{DISCUSSION}

The pathology presented by the patient in our case was uncommon, and there were no validated clinical guides for its management. Probably, the most important result of this report is the importance of rapid preoperative management (less than two hours from beginning of the symptoms to surgery) that avoided both a fatal conclusion and an extensive intestinal resection.

In the literature, a rectal prolapse with a long evolution is the predisposing factor that has been most frequently related to the pathology observed in our patient because such a prolapse induces ischemia in the neck of the hernia pouch and in the anterior rectal wall [5]. Other factors related to this pathology are the presence of abnormally-wide rectovesical/rectovaginal pouches, the existence of an underlying colorectal pathology (diverticulosis, colitis, ulceration, neoplasia, etc.), iatrogenic causes, and closed abdominal trauma [6]. Among the triggering factors, we 
must highlight all those causing an increase in the abdominal pressure (coughing, vomiting, weight lifting, pregnancy). Our patient did not present with a rectal prolapse. However, her pelvic anatomy facilitated contact with the superior rectum upon manipulation.

In most cases, a spontaneous break may occur. Some of the findings in our patient, such as the localization of the perforation in the anterior face (where the blood flow is scarce) and the difficulty with defecation (which increases the intra-abdominal pressure) caused us to suspect that a spontaneous break might also occur in our case [7]. To the contrary, the published cases report typical histological findings (ischemic areas, chronic damage, fibrosis, etc.) in patients with a spontaneous break; however, in our case, only unspecific changes were noted. Also, due to rectal lateral bending, the distance between the anus and the peritoneum was only 7-12 cm [8], allowing intraperitoneal injuries to be inflicted by the penetration of short objects. Because of all this, together with the history of manipulation during defecation, we considered rectal self-injury by digitation to be an etiologic factor.

Although cases of a primary anastomosis being performed to manage a rectal perforation have been described, because of our patient's poor condition before and during the surgery, her malnutrition, and her previous defecation problems, a colostomy was the modality of choice. In a reported series, mortality due to spontaneous perforation of the rectum was $100 \%$ when the condition was left untreated or only reduced. When the tear was only sutured, the mortality decreased to $46 \%$, and when suturing was accompanied by a colostomy, the mortality decreased to $23 \%$. Only two cases of Hartmann's procedure have been reported, and those involved no mortalities [9].

\section{CONFLICT OF INTEREST}

No potential conflict of interest relevant to this article was re- ported.

\section{REFERENCES}

1. Morris AM, Setty SP, Standage BA, Hansen PD. Acute transanal evisceration of the small bowel: report of a case and review of the literature. Dis Colon Rectum 2003;46:1280-3.

2. Corduk N, Koltuksuz U, Karabul M, Savran B, Bagci S, SariogluBuke A. A rare presentation of crush injury: transanal small bowel evisceration. Case report and review of the literature. Pediatr Surg Int 2011;27:1021-4.

3. Price NR, Soundappan SV, Sparnon AL, Cass DT. Swimming pool filter-induced transrectal evisceration in children: Australian experience. Med J Aust 2010;192:534-6.

4. Bronkhorst MW, Wilde JC, Hamming JF, Heij HA. Anorectal impalement in a pediatric patient with transanal evisceration of small bowel. J Pediatr Surg 2007;42:E23-5.

5. Furuya Y, Yasuhara H, Naka S, Yamazaki K, Yanagie H, Wakahara $\mathrm{T}$, et al. Intestinal evisceration through the anus caused by fragile rectal wall. Int J Colorectal Dis 2008;23:721-2.

6. Ellul JP, Mannion S, Khoury GA. Spontaneous rupture of the rectum with evisceration of the small intestine through the anus. Eur J Surg 1995;161:925-7.

7. Bhat S, Azad TP, Kaur M. Spontaneous perforation of rectum with evisceration of small bowel simulating intussusception. $\mathrm{N}$ Am J Med Sci 2010;2:596-7.

8. Meneu JC, Manrique A, Moreno A, Petrone P, Hernandez C, Moreno-Gonzalez M, Hidalgo M. Traumatismos de colon y recto. Madrid; Jarpyo Ed; c2005. p. 443-54.

9. Al-Abkari HA. Spontaneous rupture of the rectum with evisceration of omentum through the anus: a case report and review of the literature. Ann Saudi Med 2000;20:246-7. 